\title{
A refinement of Sándor-Tóth's inequality
}

Nicuşor Minculete ${ }^{1}$, Claudiu Pozna ${ }^{2,3}$ and Radu-Emil Precup ${ }^{4^{*}}$

* Correspondence: radu.
precup@aut.upt.ro
${ }^{4}$ Department of Automation and
Applied Informatics, Politehnica
University of Timişoara, 300223
Timişoara, Romania
Full list of author information is
available at the end of the article

\begin{abstract}
The objective of this article is to show a refinement of Sándor-Tóth's inequality related to the arithmetic functions which use unitary divisors. A new estimate of the average order of the arithmetic function given by Sándor-Tóth's inequality is suggested.
\end{abstract}

Keywords: unitary divisor, Sándor-Tóth's inequality, arithmetic function, average order

\section{Introduction}

The inequalities are important in many applications. Some attractive recent theoretical results related to inequalities include Jensen type inequalities [1], the general variational inequality problem [2], delay integral inequalities [3], inequalities that involve higher-order partial derivatives [4] or harmonic quasiconformal mappings [5]. We focus on inequalities that employ arithmetic functions based on the divisors of positive integers.

Among the divisors of a positive integer identifying a particular type of divisors, namely, the unitary divisors. But, first we present a brief history of this.

In [6], Vaidyanathaswamy introduced the notion of block-factor in the following way: a divisor $d$ of $n$ is a block-factor when $\left(d, \frac{n}{d}\right)=1$, so the greatest common divisor of $d$ and $\frac{n}{d}$ is 1 . Later Cohen gave in [7] another terminology for block-factor which is currently referred to as unitary divisor.

For example, 4 is a unitary divisor of 12 , because $\left(4, \frac{12}{4}\right)=(4,3)=1$, but 2 is not a unitary divisor of 12 , because $\left(2, \frac{12}{2}\right)=(2,6)=2 \neq 1$.

We observe that for a prime power $p^{a}$, the unitary divisors are 1 and $p^{a}$.

Let $\tau^{*}(n)$ denotes the number of unitary divisors of $n$, which is, in fact the number of the square free of $n$. Let $\sigma_{k}^{*}(n)$ denotes the sum of $k$ th powers of the unitary divisors of $n$.

If $n=\prod_{i=1}^{r} p_{i}^{a_{i}}$ is the prime factorization of $n>1$, where $p_{i}$ are distinct primes and $a_{i} \geq 1$ for all $i=1, \ldots, r$, then

(c) 2012 Minculete et al; licensee Springer. This is an Open Access article distributed under the terms of the Creative Commons Attribution License (http://creativecommons.org/licenses/by/2.0), which permits unrestricted use, distribution, and reproduction in any medium, provided the original work is properly cited. 


$$
\sigma_{k}^{*}(n)=\prod_{i=1}^{r}\left(p_{i}^{k a_{i}}+1\right)
$$

and

$$
\tau^{*}(n)=2^{r},
$$

where $r$ is the number of distinct prime factors of $n$.

We note by $\gamma(n)$ the largest divisor of $n$, which is squarefree, thus

$$
\gamma(n)=p_{1} p_{2} \cdots p_{r}
$$

and $\gamma(1)=1$, by convention.

In $[8,9]$, Sándor and Tóth proved the inequality

$$
\frac{n^{k}+1}{2} \geq \frac{\sigma_{k}^{*}(n)}{\tau^{*}(n)} \geq \sqrt{n^{k}}
$$

for any $n \geq 1$ and $k \geq 0$.

This article aims two goals, a theoretical goal and an application goal. First, a refinement of this inequality is offered to fulfil the theoretical goal. Second, the Matlab mathematical software is used to analyze the behavior of the difference

$$
\Delta_{k}(n)=\frac{\sigma_{k}^{*}(n)}{\tau^{*}(n)}-\sqrt{n^{k}}
$$

in the case $k=1$ and to fulfil the application goal.

Our new theoretical results are presented in the following section as a new inequality expressed as an improvement of (4). An application in terms of the Matlab-based solving of (5) is included as well. The conclusions are highlighted in Section 3.

\section{Main results}

Lemma 2.1. For any $n \geq 1$ and $k \geq 0$, the following inequality holds:

$$
\frac{\sigma_{k}^{*}(n)}{\tau^{*}(n)} \geq\left[\frac{n}{\gamma(n)}\right]^{k}
$$

Proof. For $n=1$, we obtain $\frac{\sigma_{k}^{*}(1)}{\tau^{*}(1)}=1=\left[\frac{1}{\gamma(1)}\right]^{k}$.

For $n>1$ the canonical form of $n$ is $n=\prod_{i=1}^{r} p_{i}^{a_{i}}$.

Using the inequality

$$
p^{k(a+1)}+p^{k} \geq 2 p^{k a}
$$

which is true, for any prime number $p$ for any $a \geq 0$ and $k \geq 0$.

Therefore, we derive the result

$$
\prod_{i=1}^{r}\left(p_{i}^{k\left(a_{i}+1\right)}+p_{i}^{k}\right) \geq 2^{r} \prod_{i=1}^{r} p_{i}^{k a_{i}}
$$

which implies the inequality

$$
\gamma^{k}(n) \sigma_{k}^{*}(n) \geq \tau^{*}(n) \cdot n^{k}
$$


Consequently, the relation (6) is true.

口

We will find next, an expression of $n$ for which the Sándor-Tóth inequality can be refined.

Theorem 2.2. For any $n=\prod_{i=1}^{r} p_{i}^{a_{i}}$ with $a_{i} \geq 2$ for all $i=1, \ldots, n$, the following inequality holds:

$$
\frac{\sigma_{k}^{*}(n)}{\tau^{*}(n)} \geq\left[\frac{n}{\gamma(n)}\right]^{k} \geq \sqrt{n^{k}}
$$

where $k \geq 1$.

Proof. We first prove that

$$
\frac{n}{\gamma(n)} \geq \sqrt{n},
$$

for $n=\prod_{p \mid n} p^{a}$, with $a \geq 2$.

Since $p^{a-1} \geq p^{a / 2}$, for any prime number $p$ and for any $a \geq 2$, it follows that

$$
\prod_{p \mid n} p^{a-1} \geq \sqrt{\prod_{p \mid n} p^{a}}
$$

which is equivalent to

$$
\frac{n}{\gamma(n)} \geq \sqrt{n} \text {. }
$$

The combination of Lemma 2.1 and of the inequality (8) results finally in the inequality (7).

口

Remark 2.1. (a) If $n$ is squarefree, then the relation

$$
\frac{\sigma_{k}^{*}(n)}{\tau^{*}(n)} \geq \sqrt{n^{k}} \geq\left[\frac{n}{\gamma(n)}\right]^{k},
$$

is true for any $n \geq 1$ and $k \geq 0$.

(b) The inequality (7) can be expressed in terms of

$$
\frac{\sigma_{k}^{*}\left(n^{2}\right)}{\tau^{*}(n)} \geq\left[\frac{n^{2}}{\gamma(n)}\right]^{k} \geq n^{k},
$$

for any $n \geq 1$ and $k \geq 0$.

Lemma 2.3. For any $n \geq 1$ and $x_{i} \geq y_{i}>1$, for all $i=1, \ldots, n$, we have

$$
\prod_{i=1}^{n}\left(x_{i} y_{i}+1\right) \geq 2^{n-1}\left(\prod_{i=1}^{n} x_{i}+\prod_{i=1}^{n} y_{i}\right) \text {. }
$$

Proof. The mathematical induction is applied to prove this lemma. For $n=1$, we obtain

$$
x_{1} y_{1}+1 \geq x_{1}+y_{1},
$$

which is true because it is equivalent to the inequality $\left(x_{1}-1\right)\left(y_{1}-1\right) \geq 0$. 
We consider that the inequality (11) is true for $n$ and we will prove that it is also true for $n+1$, thus:

$$
\begin{gathered}
\prod_{i=1}^{n+1}\left(x_{i} y_{i}+1\right)=\left(x_{n+1} y_{n+1}+1\right) \prod_{i=1}^{n}\left(x_{i} y_{i}+1\right) \geq \\
\geq 2^{n-1}\left(x_{n+1} y_{n+1}+1\right)\left(\prod_{i=1}^{n} x_{i}+\prod_{i=1}^{n} y_{i}\right) .
\end{gathered}
$$

Let us consider $\prod_{i=1}^{n} x_{i}=x$ and $\prod_{i=1}^{n} y_{i}=y$, with $x \geq y$. We will prove that

$$
\left(x_{n+1} y_{n+1}+1\right)(x+y) \geq 2\left(x_{n+1} x+y_{n+1} y\right),
$$

which is equivalent to the inequality

$$
\left(x_{n+1} x-y\right)\left(y_{n+1}-1\right)+\left(y_{n+1} y-x\right)\left(x_{n+1}-1\right) \geq 0 .
$$

But $x_{n+1}-1 \geq y_{n+1}-1 \geq 0$, which means that the inequality (14) becomes, by minorization,

$$
\begin{gathered}
\left(y_{n+1}-1\right)\left(x_{n+1} x-y+y_{n+1} y-x\right)= \\
\left(y_{n+1}-1\right)\left[x\left(x_{n+1}-1\right)+y\left(y_{n+1}-1\right)\right] \geq 0,
\end{gathered}
$$

which is true.

The combination of the inequalities (12) and (13) leads to the result.

$$
\prod_{i=1}^{n+1}\left(x_{i} y_{i}+1\right) \geq 2^{n}\left(\prod_{i=1}^{n+1} x_{i}+\prod_{i=1}^{n+1} y_{i}\right) .
$$

According to the principle of mathematical induction, the inequality (11) is true. 口

Another improvement of Sándor-Tóth's inequality is presented as follows in terms of Theorem 2.4.

Theorem 2.4. For any $n \geq 1$ and $k \geq 1$ there the following inequality holds:

$$
\frac{n^{k}+1}{2} \geq \frac{\sigma_{k}^{*}(n)}{\tau^{*}(n)} \geq \frac{1}{2}\left[\frac{n^{k}}{\sqrt{\gamma(n)}}+\sqrt{\gamma(n)}\right] \geq \sqrt{n^{k}} .
$$

Proof. The mathematical induction is also applied to prove this theorem. For $n=1$, we have the equality in relation (15). If $n=p_{1}^{a_{1}} \ldots p_{r}^{a_{r}}>1$, then, from Lemma 2.3, we have

$$
\begin{aligned}
& \frac{\sigma_{k}^{*}(n)}{\tau^{*}(n)}=\prod_{i=1}^{r}\left(\frac{p_{i}^{a_{i} k}+1}{2}\right)=\frac{1}{2^{r}} \prod_{i=1}^{r}\left(p_{i}^{a_{i} k-\frac{1}{2}} \cdot p_{i}^{\frac{1}{2}}+1\right) \geq \\
& \geq \frac{1}{2}\left(\prod_{i=1}^{r} p_{i}^{a_{i} k-\frac{1}{2}}+\prod_{i=1}^{r} p_{i}^{\frac{1}{2}}\right)=\frac{1}{2}\left[\frac{n^{k}}{\sqrt{\gamma(n)}}+\sqrt{\gamma(n)}\right] .
\end{aligned}
$$

In fact, the inequality

$$
\frac{1}{2}\left[\frac{n^{k}}{\sqrt{\gamma(n)}}+\sqrt{\gamma(n)}\right] \geq \sqrt{n^{k}} .
$$


is immediate because the arithmetic mean is greater than the geometric mean.

Let $d$ be a divisor of $n$, then

$$
\left(n^{k}-d^{k}\right)\left(1-\frac{1}{d^{k}}\right) \geq 0
$$

so

$$
n^{k}+1 \geq d^{k}+\left(\frac{n}{d}\right)^{k}
$$

The calculation of the sum for all divisors of $n$ results in the relation

$$
\left(n^{k}+1\right) \tau^{*}(n) \geq 2 \sigma_{k}^{*}(n),
$$

which is equivalent to the inequality

$$
\frac{n^{k}+1}{2} \geq \frac{\sigma_{k}^{*}(n)}{\tau^{*}(n)} \text {. }
$$

Therefore the proof is complete.

口

Corollary 2.5. For any $n \geq 1$, the inequality

$$
\frac{n^{k}+1}{2} \geq \frac{\sigma_{k}^{*}(n)}{\tau^{*}(n)} \geq \frac{1}{2}\left[\frac{n^{k}}{\gamma(n)}+\gamma(n)\right] \geq \sqrt{n^{k}},
$$

holds for any $k \geq 2$.

Proof. Applying Theorem 2.4, we obtain

$$
\frac{n^{k}+1}{2} \geq \frac{\sigma_{k}^{*}(n)}{\tau^{*}(n)} \geq \frac{1}{2}\left[\frac{n^{k}}{\sqrt{\gamma(n)}}+\sqrt{\gamma(n)}\right] .
$$

We apply the next inequality

$$
\frac{n^{k}}{\sqrt{\gamma(n)}}+\sqrt{\gamma(n)} \geq \frac{n^{k}}{\gamma(n)}+\gamma(n)
$$

which is equivalent to

$$
(\sqrt{\gamma(n)}-1)\left(n^{k}-\gamma(n) \sqrt{\gamma(n)}\right) \geq 0,
$$

and this is true for any $n \geq 1$ and $k \geq 2$.

Since the arithmetic mean is greater than the geometric mean, it follows that

$$
\frac{1}{2}\left[\frac{n^{k}}{\gamma(n)}+\gamma(n)\right] \geq \sqrt{n^{k}}
$$

Combining relations (17), (18), and (19), we derive the inequality (16).

$\square$

Remark 2.2. The inequality (15) is an improvement of Sándor-Tóth's inequality, and we obtain the relation 


$$
\frac{\left(\sqrt{n^{k}}-1\right)^{2}}{2} \geq \Delta_{k}(n) \geq \frac{\left[\sqrt{n^{k}}-\sqrt{\gamma(n)}\right]^{2}}{2 \sqrt{\gamma(n)}} \geq 0,
$$

for every $n \geq 1$ and $k \geq 1$.

Using the Matlab mathematical software we represent as follows the functions $f(n)=\frac{(\sqrt{n}-1)^{2}}{2}, \Delta_{1}(n)$ and $g(n)=\frac{[\sqrt{n}-\sqrt{\gamma(n)}]^{2}}{2 \sqrt{\gamma(n)}}$ in the same Cartesian coordinate system for $n \leq 10,000$, when $\Delta_{1}(n)$ is a positive integer number (see Figure 1 ).

Theorem 2.6. For any $n \geq 1$ and $k \geq 1$, the inequality

$$
\Delta_{k}(n)=\frac{\sigma_{k}^{*}(n)}{\tau^{*}(n)}-\sqrt{n^{k}} \leq \frac{\tau(n)}{\tau^{*}(n)}\left[\frac{\sigma_{k}(n)}{\tau(n)}-\sqrt{n^{k}}\right],
$$

holds, where $\sigma_{k}(n)$ is the sum of kth powers of the divisors of $n$ and $\tau(n)$ is the number of divisors of $n$.

Proof. Using the identity of Dinghas [10], we prove the Radó inequality [11]

$$
n\left(A_{n}-G_{n}\right) \geq(n-1)\left(A_{n-1}-G_{n-1}\right),
$$

where $A_{k}$ is the arithmetic mean and $G_{k}$ is the geometric mean of $k$ numbers of $a_{1}$, $a_{2}, \ldots, a_{n}(k \leq n)$.

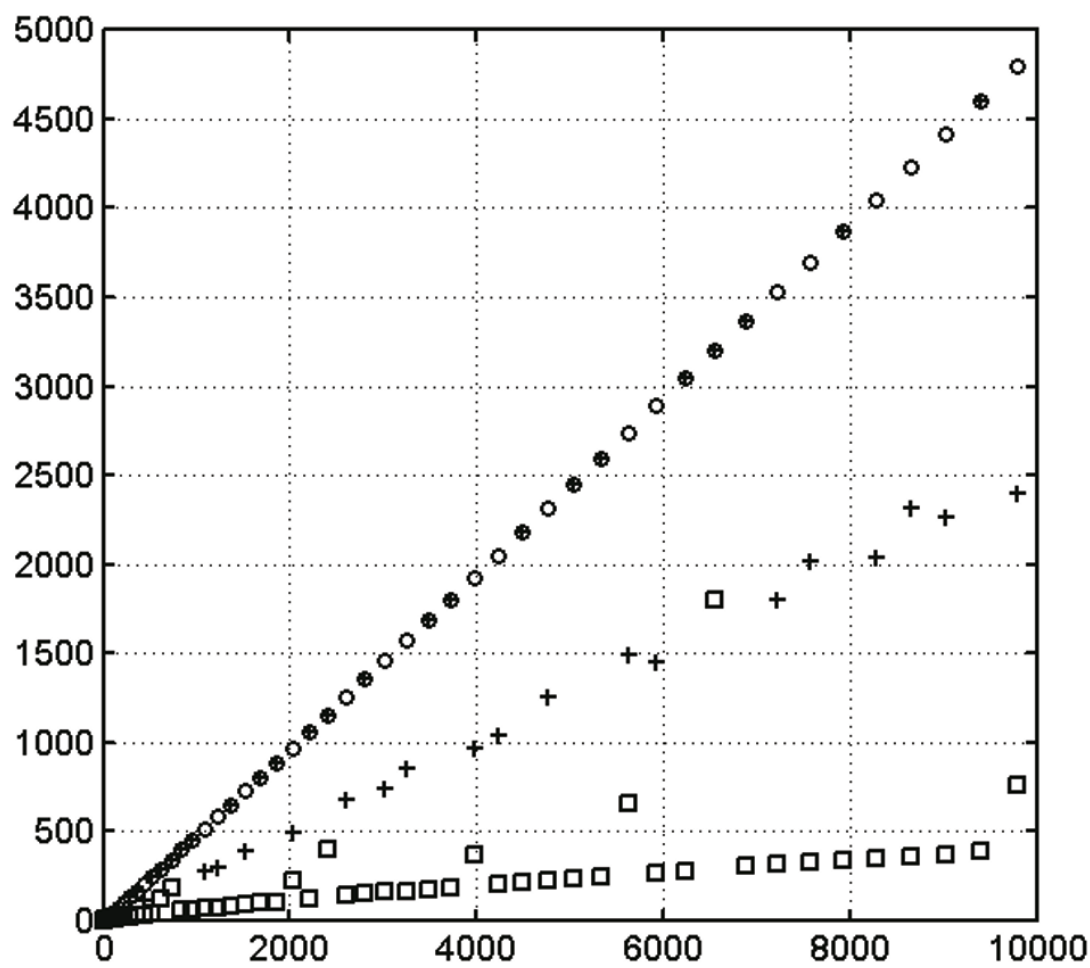

Figure 1 Variations of functions $f, \Delta_{1}$ and $g$ versus $\boldsymbol{n}$. The symbols $0_{1}+, \square$ are used to represent the functions $f, \Delta_{1}$ and $g$, respectively. 
Therefore, from the inequality (22), for $n \geq m$, we derive the result

$$
n\left(A_{n}-G_{n}\right) \geq m\left(A_{m}-G_{m}\right) .
$$

We consider that $d_{1}^{*}, d_{2}^{*}, \ldots, d_{s}^{*}$ are the unitary divisors of $n$, and $d_{1}, d_{2}, \ldots, d_{s}, d_{s+1}, \ldots$, $d_{t}(t \geq s)$ are all divisors of $n$, where $d_{i}=d_{i}^{*}(i=\overline{1, s})$. It follows, from the inequality (23), that

$$
t\left(\frac{\sum_{i=1}^{t} d_{i}^{k}}{t}-\left(\prod_{i=1}^{t} d_{i}^{k}\right)^{1 / t}\right) \geq s\left(\frac{\sum_{i=1}^{s} d_{i}^{* k}}{s}-\left(\prod_{i=1}^{s} d_{i}^{* k}\right)^{1 / s}\right)
$$

so

$$
\tau(n)\left(\frac{\sigma_{k}(n)}{\tau(n)}-\sqrt{n^{k}}\right) \geq \tau^{*}(n)\left(\frac{\sigma_{k}^{*}(n)}{\tau^{*}(n)}-\sqrt{n^{k}}\right)
$$

because

$$
\left(\prod_{i=1}^{t} d_{i}\right)^{1 / t}=\left(\prod_{i=1}^{s} d_{i}^{*}\right)^{1 / s}=\sqrt{n}
$$

Consequently, the inequality (21) is proved.

$\square$

Theorem 2.7. For $n \geq 1$ and $k \geq 0$, there is the inequality

$$
\begin{gathered}
\frac{1}{2 n \tau^{*}(n)}\left[\sigma_{2 k}^{*}(n)-\left(\frac{\sigma_{k}^{*}(n)}{\tau^{*}(n)}\right)^{2}\right] \leq \frac{\sigma_{k}^{*}(n)}{\tau^{*}(n)}-\sqrt{n^{k}} \leq \\
\frac{1}{2 \tau^{*}(n)}\left[\sigma_{2 k}^{*}(n)-\left(\frac{\sigma_{k}^{*}(n)}{\tau^{*}(n)}\right)^{2}\right] .
\end{gathered}
$$

Proof. Cartwright and Field proposed in [12] the inequality Let $0<m=\min \left\{x_{1}, x_{2}, \ldots, x_{n}\right\}$ and let $M=\max \left\{x_{1}, x_{2}, \ldots, x_{n}\right\}$.

Then

$$
\begin{gathered}
\frac{1}{2 M} \sum_{i=1}^{n} \alpha_{i}\left(x_{i}-\sum_{k=1}^{n} \alpha_{k} x_{k}\right)^{2} \leq \sum_{i=1}^{n} \alpha_{i} x_{i}-\prod_{i=1}^{n} x_{i}^{\alpha_{i}} \leq \\
\leq \frac{1}{2 m} \sum_{i=1}^{n} \alpha_{i}\left(x_{i}-\sum_{k=1}^{n} \alpha_{k} x_{k}\right)^{2}
\end{gathered}
$$

where $\sum_{i=1}^{n} \alpha_{i}=1$.

If $d_{1}, d_{2}, \ldots, d_{s}$ are the unitary divisors of $n$, we take $\alpha_{i}=\frac{1}{s}$ and $x_{i}=d_{i}^{k}$ in inequality (25).

Therefore, we have $m=1, M=n$ and $s=\tau^{*}(n)$, and the inequality (25) becomes:

$$
\begin{gathered}
\frac{1}{2 n s} \sum_{i=1}^{s}\left(d_{i}^{k}-\frac{\sigma_{k}^{*}(n)}{\tau^{*}(n)}\right)^{2} \leq \frac{\sigma_{k}^{*}(n)}{\tau^{*}(n)}-\sqrt{n^{k}} \leq \\
\leq \frac{1}{2 s} \sum_{i=1}^{s}\left(d_{i}^{k}-\frac{\sigma_{k}^{*}(n)}{\tau^{*}(n)}\right)^{2} .
\end{gathered}
$$


Conducting simple calculations and accounting for

$$
\left(\prod_{i=1}^{s} d_{i}^{k}\right)^{1 / s}=\left(\prod_{i=1}^{s} d_{i}\right)^{\frac{k}{S}}=\left(n^{\frac{S}{2}}\right)^{\frac{k}{s}}=n^{\frac{k}{2}},
$$

we observe that this inequality is equivalent to the inequality (24).

$\square$

Remark 2.3. (a) The inequality (24) is another improvement of Sándor-Tóth's inequality. We also obtain the following result:

$$
\begin{gathered}
\sigma_{2 k}^{*}(n)-\left(\frac{\sigma_{k}^{*}(n)}{\tau^{*}(n)}\right)^{2} \leq 2 n \tau^{*}(n) \Delta_{k}(n) \leq \\
\leq n\left[\sigma_{2 k}^{*}(n)-\left(\frac{\sigma_{k}^{*}(n)}{\tau^{*}(n)}\right)^{2}\right],
\end{gathered}
$$

(b) Using the Matlab mathematical software we find the following characterization: if $n$ is the square of an odd integer, then $\Delta_{k}(n)$ is a positive integer. This fact proved relatively easily taking into account that

$$
\frac{\sigma_{k}^{*}(n)}{\tau^{*}(n)}=\prod_{p \mid n}\left(\frac{p^{2 k a}+1}{2}\right)
$$

is a positive integer because $p$ is odd prime number.

For example, if $n \in\{11025,27225,65029\}$, then $\Delta_{1}(n) \in\{1520,3800,9170\}$.

We find next an estimate of the average order of the function $\Delta_{1}(n)$.

The average order of the function $\Delta_{1}(n)$ is the sum

$$
\Delta(x)=\sum_{n \leq x} \Delta_{1}(n)
$$

Theorem 2.8. For all $x \geq 1$, we have

$$
\begin{gathered}
\frac{\pi^{2} x \sqrt[3]{x^{2}}}{40 \zeta(3)}-\frac{2}{3} x \sqrt{x}+O\left(x^{2 / 3} \log ^{2 / 3} x\right) \leq \Delta(x) \leq \\
\leq \frac{\pi^{2} x^{2}}{24 \zeta(3)}-\frac{2}{3} x \sqrt{x}+O\left(x \log ^{2 / 3} x\right)
\end{gathered}
$$

where $\varsigma$ is the Riemann zeta function, $\varsigma(3)$ is Apéry's constant with $\varsigma(3)=$ $1.2020569032 . .$. and $O$ is the symbol of Landau.

Proof. Sándor and Kovács offered recently [13] a result related to the function $\tau(n)$, which is the number of divisors of $n$, namely,

$$
\tau(n)<4 \sqrt[3]{n},
$$

for all $n \geq 1$.

But, the number of divisors of $n$ is greater than the number of unitary divisors of $n$, so

$$
2 \leq \tau^{*}(n)<4 \sqrt[3]{n}
$$

for all $n \geq 2$. 
Sitaramachandrarao and Surynarayana pointed out in [8] the following estimate of $\sigma^{*}(n)$ :

$$
\sum_{n \leq x} \sigma^{*}(n)=\frac{\pi^{2} x^{2}}{12 \zeta(3)}+O\left(x \log ^{2 / 3} x\right)
$$

Nathanson proved in [14] that if $x$ and $y$ are real numbers with $y<[x]$, and $f(t)$ if is a nonnegative monotonic function on $[y, x]$, then

$$
\left|\sum_{\gamma<n \leq x} f(n)-\int_{\gamma}^{x} f(t) d t\right| \leq \max \{f(x), f(\gamma)\} .
$$

For $f(n)=\sqrt{n}$, we find the average order of $\sqrt{n}$, thus

$$
\sum_{n \leq x} \sqrt{n}=\frac{2}{3} x \sqrt{x}+O(\sqrt{x})
$$

We will calculate the sum $\sum_{n \leq x} \frac{\sigma^{*}(n)}{\sqrt[3]{n}}$ using the theorem of partial summation [14], thus

$$
\sum_{n \leq x} f(n) g(n)=F(x) g(x)-\int_{1}^{x} F(t) g^{\prime}(t) d t
$$

where $f(n)$ and $g(n)$ are two arithmetic functions, $x \geq 2, g(t)$ is continuously differentiable on $[1, x]$, and $F(x)=\sum_{n \leq x} f(n)$.

Therefore, for $f(n)=\sigma^{*}(n), g(n)=\frac{1}{\sqrt[3]{n}}$ and $F(x)=\frac{\pi^{2} x^{2}}{12 \zeta(3)}+O\left(x \log ^{2 / 3} x\right)$ (from (29)), relation (31) results in

$$
\begin{aligned}
& \sum_{n \leq x} \frac{\sigma^{*}(n)}{\sqrt[3]{n}}=\left(\frac{\pi^{2} x^{2}}{12 \zeta(3)}+O\left(x \log ^{2 / 3} x\right)\right) \frac{1}{\sqrt[3]{x}}+ \\
& +\frac{1}{3} \int_{1}^{x}\left[\frac{\pi^{2} t^{2}}{12 \zeta(3)}+O\left(t \log ^{2 / 3} t\right)\right] \frac{1}{t^{4 / 3}} d t= \\
& =\frac{\pi^{2} x^{5 / 3}}{10 \zeta(3)}+O\left(x^{2 / 3} \log ^{2 / 3} x\right)+\frac{1}{3} \int_{1}^{x} o\left(\frac{1}{t^{1 / 3} \log ^{2 / 3} t}\right) d t \\
& =\frac{\pi^{2} x^{5 / 3}}{10 \zeta(3)}+O\left(x^{2 / 3} \log ^{2 / 3} x\right)
\end{aligned}
$$

so

$$
\sum_{n \leq x} \frac{\sigma^{*}(n)}{\sqrt[3]{n}}=\frac{\pi^{2} x \sqrt[3]{x^{2}}}{10 \zeta(3)}+O\left(x^{2 / 3} \log ^{2 / 3} x\right)
$$

Since $\Delta_{1}(n)=\frac{\sigma^{*}(n)}{\tau^{*}(n)}-\sqrt{n}$, the application of (28) leads to

$$
\begin{gathered}
\frac{1}{4} \sum_{n \leq x} \frac{\sigma^{*}(n)}{\sqrt[3]{n}}-\sum_{n \leq x} \sqrt{n} \leq \sum_{n \leq x} \Delta_{1}(x) \leq \\
\leq \frac{1}{2} \sum_{n \leq x} \sigma^{*}(n)-\sum_{n \leq x} \sqrt{n} .
\end{gathered}
$$


Summing up, the relations (29), (30), (32), and (33) lead to the fulfilment of (27).

\section{Conclusions}

This article has proposed a refinement of Sándor-Tóth's inequality, and two Matlab applications are given. Theorem 2.8 offers an approximation of the average order of $\Delta$ $(x)$. Finding the average order of $\Delta(x)$ and the average order of

$$
\Delta_{k}(x)=\sum_{n \leq x} \Delta_{k}(n) .
$$

are subjects of future research. Studying the ideas above, we can identify other refinements of Sándor-Tóth's inequality.

The future research will also focus the extension of the area of applications of our new theoretical results. Such applications include solutions to optimal control problems [15], stability analysis $[16,17]$, robotics [18], fuzzy logic $[19,20]$, difference inequalities [21] or differential equations [22], as far as positive integers are concerned.

\section{Acknowledgements}

The authors were grateful to the referee for useful comments. This study was supported by the Romanian Ministry of Education, Research and Innovation through the PNII Idei project 842/2008.

\section{Author details}

'Department of Mathematics, Dimitrie Cantemir University of Braşov, Braşov 500068, Romania ${ }^{2}$ Department of Informatics, Széchenyi István University, 9026 Györ, Hungary ${ }^{3}$ Transilvania University of Braşov, 500036 Braşov, Romania ${ }^{4}$ Department of Automation and Applied Informatics, Politehnica University of Timişoara, 300223 Timiş̧oara, Romania

\section{Authors' contributions}

NM completed the main part of this article, CP presented the ideas of this article, REP participated in some study of this article. REP made the text file and communicated the manuscript. All authors read and approved the final manuscript.

\section{Competing interests}

The authors declare that they have no competing interests.

Received: 29 August 2011 Accepted: 9 January 2012 Published: 9 January 2012

\section{References}

1. Agarwal, RP, Dragomir, SS: A survey of Jensen type inequalities for functions of selfadjoint operators in Hilbert spaces. Comput Math Appl. 59(12), 3785-3812 (2010). doi:10.1016/j.camwa.2010.04.014

2. Cho, YJE, Petrot, N: Regularization and iterative method for general variational inequality problem in Hilbert spaces. J Inequal Appl. 2011, 21 (2011). doi:10.1186/1029-242X-2011-21

3. Feng, Q, Meng, F, Zhang, Y, Zheng, Bs, Zhou, J: Some nonlinear delay integral inequalities on time scales arising in the theory of dynamics equations. J Inequal Appl. 2011, 29 (2011). doi:10.1186/1029-242X-2011-29

4. Zhao, C-J, Cheung, W-S: On some Opial-type inequalities. J Inequal Appl. 2011, 7 (2011). doi:10.1186/1029-242X-2011-7

5. Arsenovic, M, Manojlovic, V, Vuorinen, M: Hölder continuity of harmonic quasiconformal mappings. J Inequal Appl. 2011, 37 (2011). doi:10.1186/1029-242X-2011-37

6. Vaidyanathaswamy, R: The theory of multiplicative arithmetic functions. Trans Am Math Soc. 33, 579-662 (1931). doi:10.1090/S0002-9947-1931-1501607-1

7. Cohen, E: Arithmetical functions associated with the unitary divisors of an integer. Math Z. 74, 66-80 (1960). doi:10.1007/BF01180473

8. Sándor, J, Crstici, B: Handbook of Number Theory II. Springer, New York (2006)

9. Sándor, J, Tóth, L: On certain number-theoretic inequalities. Fib Quart. 28, 255-258 (1990)

10. Dinghas, A: Some identities between arithmetic means and the other elementary symmetric functions of $n$ numbers. Math Ann. 120(1), 154-157 (1948)

11. Hardy, GH, Littlewood, JE, Polya, G: Inequalities. Cambrige University Press, Cambridge (1952)

12. Cartwright, DI, Field, MJ: A refinement of the arithmetic mean-geometric mean inequality. Proc Am Math Soc. 71(1), 36-38 (1978). doi:10.1090/S0002-9939-1978-0476971-2

13. Sándor, J, Kovács, L: An inequality for the number of divisors of $n$. Octog Math Mag. 17(2), 746-749 (2009)

14. Nathanson, M: Elementary Methods in Number Theory. Springer, New York (2000)

15. Precup, R-E, Preitl, S, Rudas, IJ, Tomescu, ML, Tar, JK: Design and experiments for a class of fuzzy controled servo systems. IEEE/ASME Trans Mechatron. 13(1), 22-35 (2008)

16. Pastravanu, O, Matcovschi, M-H: Diagonal stability of interval matrices and applications. Linear Algebra Appl. 433(8-10), 1646-1658 (2010). doi:10.1016/j.laa.2010.06.016 
17. Park, C, Jang, SY, Lee, JR, Yun, D: On the stability of an AQCQ-functional equation in random normed spaces. J Inequal Appl. 2011, 34 (2011). doi:10.1186/1029-242X-2011-34

18. Sekiyama, K, Ito, M, Fukuda, T, Suzuki, T, Yamashita, K: An adaptive muscular force generation mechanism based on prior information of handling object. J Adv Comput Intell Intell Inf. 13(3), 222-229 (2009)

19. Mester, G: Obstacle-slope avoidance and velocity control of wheeled mobile robots using fuzzy reasoning. Proc 13th IEEE International Conference on Intelligent Engineering Systems (INES 2009). pp. 245-249.Barbados (2009)

20. Sram, N, Takacs, M: Minnesota code: A neuro-fuzzy-based decision tuning. Proc 15th IEEE International Conference on Intelligent Engineering Systems (INES 2011). pp. 191-195.Poprad, Slovakia (2011)

21. Li, D, Long, S, Wang, X: Difference inequality for stability of impulsive difference equations with distributed delays. J Inequal Appl. 2011, 8 (2011). doi:10.1186/1029-242X-2011-8

22. Mir, U, Merghem-Boulahi, L, Gaiti, D: A new framework for spectrum sharing based on multiagent systems and Petri nets. Int J Artif Intell. 6(S11), 144-160 (2011)

doi:10.1186/1029-242X-2012-4

Cite this article as: Minculete et al:: A refinement of Sándor-Tóth's inequality. Journal of Inequalities and Applications 2012 2012:4.

\section{Submit your manuscript to a SpringerOpen ${ }^{\circ}$} journal and benefit from:

- Convenient online submission

- Rigorous peer review

- Immediate publication on acceptance

- Open access: articles freely available online

- High visibility within the field

- Retaining the copyright to your article

Submit your next manuscript at $\gg$ springeropen.com 